\title{
Paraganglioma nasal: relato de caso
}

\author{
Mauren P. Rocha', Andréa M. Campagnolo', \\ Vanessa S. Macedo ${ }^{1,}$ Fabiana B. Scarton', \\ Henrique P. Rocha ${ }^{3}$, Gabriel Kubl
}

\section{Nasal paraganglioma: a case report}

Palavras-chave: paraganglioma nasal, cavidade nasal, tumor nasal.

Key words: nasal paraganglioma, nasal cavity, nasal tumour.

\section{Resumo / Summary}

$\mathbf{P}$ paragangliomas da cabeça e pescoço são bastante raros. Os tipos mais freqüentemente encontrados são o carotídeo, o júgulo-timpânico e o vagal. Paragangliomas do nariz e dos seios paranasais são muito infreqüentes. Os autores descrevem um caso de paraganglioma nasal em um paciente do sexo masculino, com 45 anos de idade, que foi submetido à exérese da lesão, e relatam os achados clínicos, critérios diagnósticos, tratamento, prognóstico e revisão da literatura. Consideramos importante o relato deste caso em vista da raridade da localização nasal dos paragangliomas.

\begin{abstract}
- aragangliomas arise from paraganglionic tissue of the autonomic nervous system. Paragangliomas of the head and neck region are rare. The most common sites of origin of this neoplasm in the head and neck region are the carotid body, the jugular bulb and the vagal body. Paragangliomas of the nose and paranasal sinuses are very uncommon. The authors referred about one case of nasal paraganglioma in a 45-year-old male patient, who was submitted to surgical excision, and discuss the clinical findings, diagnostic criteria, treatment, prognosis and literature review. The importance of reporting this case refers to the rarity of paragangliomas located in the nasal cavity and paranasal sinuses.
\end{abstract}

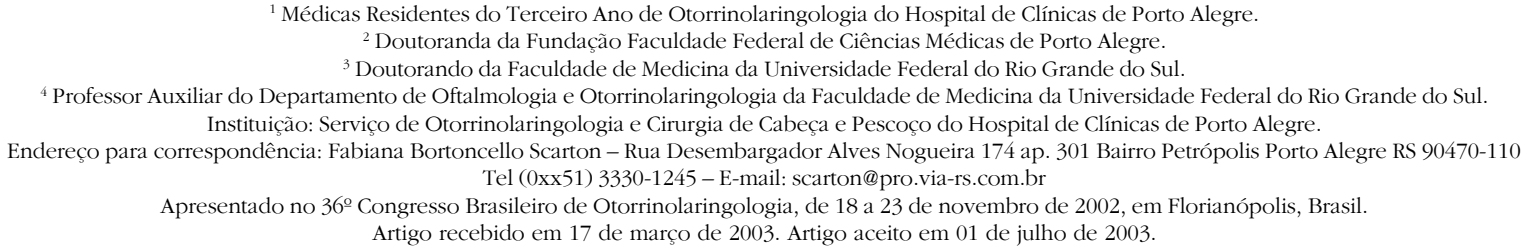

${ }^{1}$ Médicas Residentes do Terceiro Ano de Otorrinolaringologia do Hospital de Clínicas de Porto Alegre.

${ }^{2}$ Doutoranda da Fundação Faculdade Federal de Ciências Médicas de Porto Alegre.

${ }^{3}$ Doutorando da Faculdade de Medicina da Universidade Federal do Rio Grande do Sul.

${ }^{4}$ Professor Auxiliar do Departamento de Oftalmologia e Otorrinolaringologia da Faculdade de Medicina da Universidade Federal do Rio Grande do Sul. Instituição: Serviço de Otorrinolaringologia e Cirurgia de Cabeça e Pescoço do Hospital de Clínicas de Porto Alegre.

Endereço para correspondência: Fabiana Bortoncello Scarton - Rua Desembargador Alves Nogueira 174 ap. 301 Bairro Petrópolis Porto Alegre RS $90470-110$ Tel (0xx51) 3330-1245 - E-mail: scarton@pro.via-rs.com.br

Apresentado no $36^{\circ}$ Congresso Brasileiro de Otorrinolaringologia, de 18 a 23 de novembro de 2002, em Florianópolis, Brasil

Artigo recebido em 17 de março de 2003. Artigo aceito em 01 de julho de 2003. 


\section{INTRODUÇÃO}

Paragangliomas são neoplasias que surgem a partir do tecido paragangliônico do sistema nervoso autônomo. Os paragânglios apresentam origem neuroectodérmica e contêm dois tipos celulares: células tipo I, contendo grânulos de catecolaminas, e tipo II, células de sustentação, semelhantes às células de Schwann, e que circundam as de tipo $I^{1}$.

Os paragânglios estão amplamente distribuídos no corpo humano, tendo sido encontrados nos pulmões, coração, mediastino, trato gastrointestinal, retroperitônio e bexiga. $\mathrm{Na}$ cabeça e pescoço, observou-se sua presença na traquéia, língua, laringe, hipófise, glândula pineal e órbita. Mas, apesar desses achados, as localizações mais freqüentes dos paragangliomas são no corpo carotídeo, no corpo jugular, ao longo do nervo glossofaríngeo e seu ramo timpânico, e no nervo vago, especialmente próximo ao seu gânglio nodoso ${ }^{2}$.

Observamos um caso de paraganglioma nasal em um paciente masculino de 45 anos e decidimos relatá-lo devido à raridade da apresentação clínica nesta localização.

\section{REVISÃO DA LITERATURA}

Os paragangliomas da cabeça e pescoço são bastante raros. A incidência dessas neoplasias é de 0,0012\% $\%^{3}$. Em geral, são tumores sintomáticos que podem ser clinicamente confundidos com outras lesões benignas ou malignas ${ }^{3}$. Não são incomuns os casos de confusão entre paraganglioma e tumor glômico, pois, apesar de serem imuno e histologicamente distintos, ambos apresentam intensa vascularização ${ }^{4}$. Os tipos mais freqüentemente encontrados são o carotídeo, o júgulo-timpânico e o vagal5.
Não há conhecimento definitivo acerca do local de origem dos paragangliomas nasais. Muitos autores sugeriram que há tecido paragangliônico na fossa pterigopalatina baseando-se no fato de que os paragânglios mantêm íntima relação com artérias e nervos cranianos. Entretanto, dos casos previamente relatados na literatura, a maioria foi encontrada na região da concha média ou seio etmoidal ${ }^{1}$.

\section{APRESENTAÇÃO DO CASO CLÍNICO}

J.C, com 45 anos de idade, do sexo masculino, de cor branca, procurou atendimento queixando-se de obstrução nasal há um ano. Referia, ainda, secreção nasal crônica com períodos de rinorréia purulenta. Não apresentava sangramento, dor ou edema facial.

Ao exame otorrinolaringológico, a rinoscopia anterior evidenciava uma massa polipóide na fossa nasal direita. Na nasofibroscopia, observou-se lesão na fossa nasal direita que obstruía o coano ipsilateral. A tomografia computadorizada mostrava uma lesão com densidade de partes moles na cavidade nasal direita (Figura 1). Foi realizada biópsia incisional sob anestesia local que demonstrou paraganglioma nasal. Após embolização prévia da artéria maxilar por meio de arteriografia (Figura 2), o paciente foi submetido à cirurgia, com congelação no trans-operatório, em 16/01/2002, quando realizou-se a excisão total da lesão. Tanto o exame anátomopatológico de congelação como o definitivo evidenciaram paraganglioma nasal. Não houve complicações no pósoperatório imediato e tardio. Decorridos cinco meses da cirurgia, o paciente encontra-se assintomático e em acompanhamento ambulatorial, não apresentando evidências de recidiva até o momento.
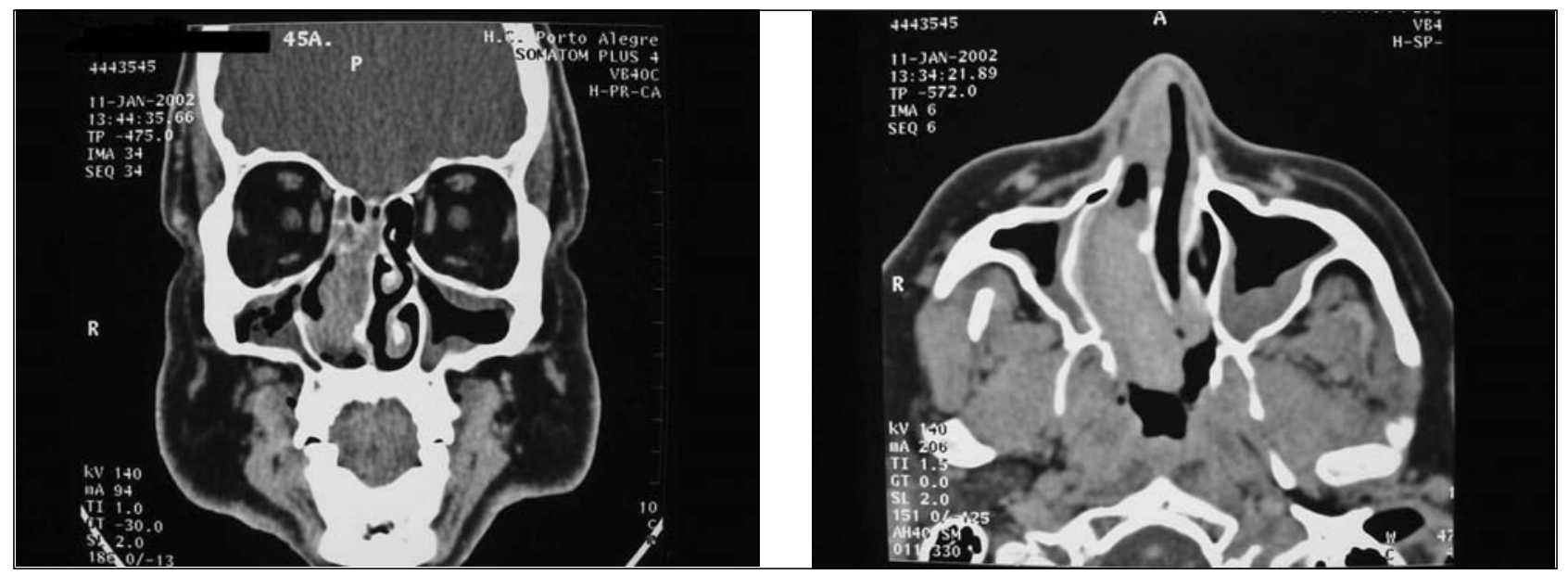

Figura 1. Dois cortes da tomografia computadorizada do paciente J.C. mostrando uma lesão com densidade de partes moles na cavidade nasal direita. 


\section{DISCUSSÃO}

Paragangliomas do nariz e dos seios paranasais são muito infreqüentes. Conforme estudo que reuniu 73 casos de paragangliomas da cabeça e pescoço, apenas 3 eram do tipo nasal e paranasal ${ }^{2}$. Uma revisão atual da literatura demonstrou apenas 22 casos de paragangliomas nessa área anatômica ${ }^{1}$.

O paraganglioma nasal é uma neoplasia de crescimento lento, cujo intervalo de tempo entre o início dos sintomas e o estabelecimento do diagnóstico pode ser de dois anos ou mais ${ }^{5}$. Há uma tendência natural bem definida para multicentricidade. Muitos tumores sincrônicos são des- cobertos incidentalmente durante a arteriografia. Normalmente, a incidência de bilateralidade e multicentricidade destes tumores é de 3\%, e aumenta para 26\% nos pacientes com história familiar positiva, mostrando a existência de uma predisposição familiar ${ }^{6}$.

As manifestações clínicas são episódios recorrentes de epistaxe leve a profusa, rinorréia, obstrução nasal e edema facial, que pode estar acompanhado de visão borrada ${ }^{3,5}$. Em geral, a neoplasia apresenta-se como uma massa polipóide aderida à parede lateral da fossa nasal ou na parte superior do teto da rinofaringe ${ }^{3}$. Em alguns casos, há extensão do paraganglioma para os seios paranasais, com erosão das paredes ósseas 5 .
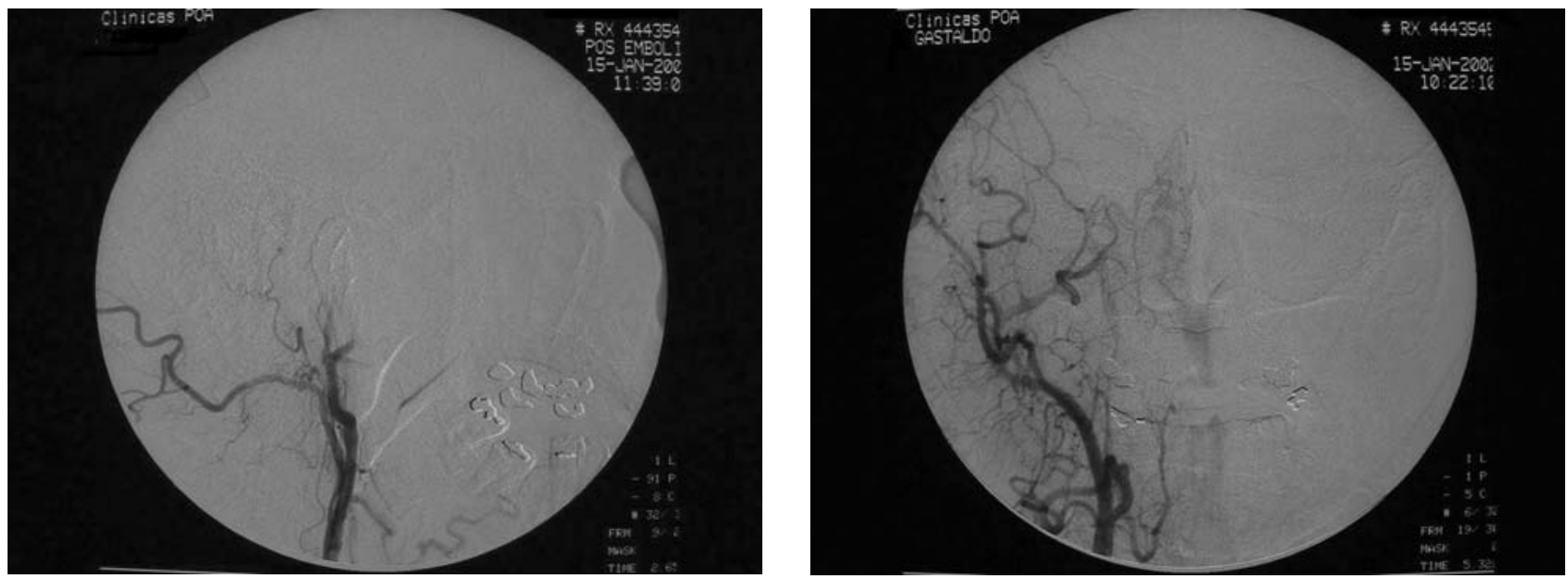

Figura 2. Cortes da arteriografia pré e pós-embolização da artéria maxilar.
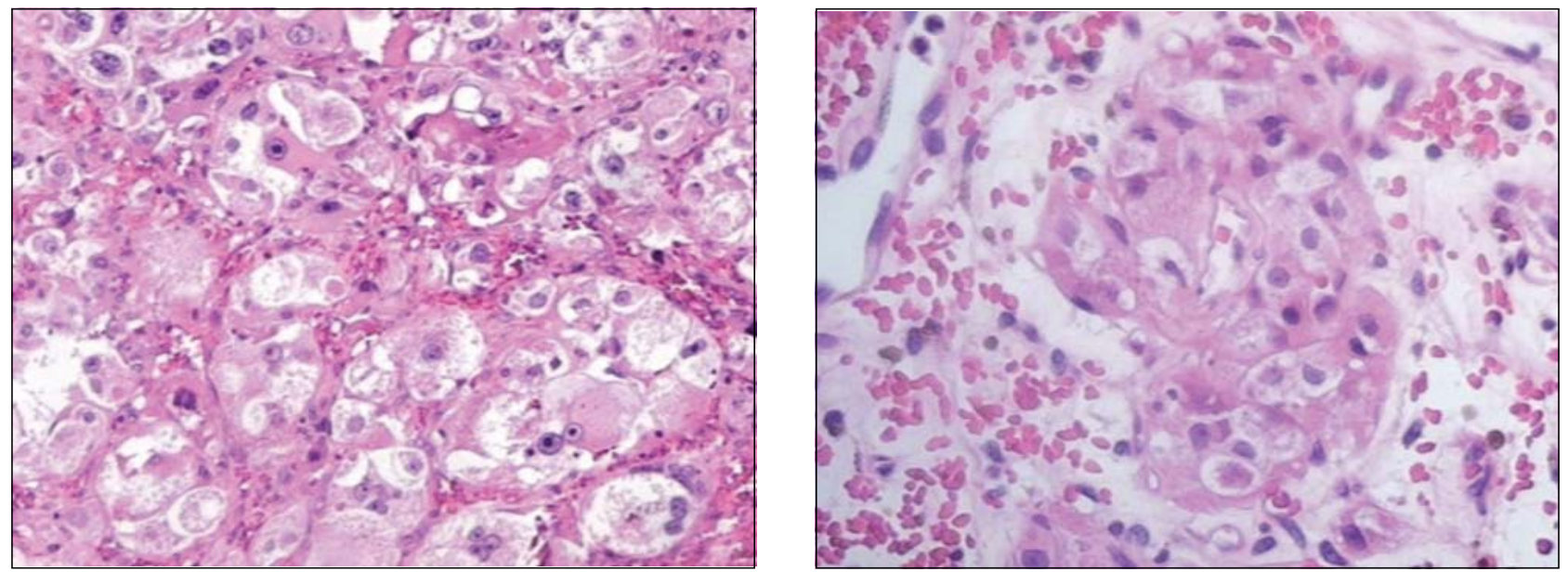

Figura 3. Cortes histológicos do paraganglioma nasal mostrando células epitelióides, que formam ninhos, chamados zellballen, separados por uma rede de capilares, corados em HE, com 50X e 100X. 
Ao exame macroscópico, os paragangliomas são lesões firmes, com coloração cinza à rosada, e com aparência encapsulada ${ }^{3}$. À histologia, essas neoplasias são caracteristicamente compostas por células epitelióides, com núcleos arredondados e citoplasma eosinofílico, que formam ninhos, chamados zellballen, separados por uma rede de capilares

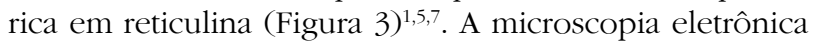
evidenciou a presença de grânulos citoplasmáticos neurossecretores no citoplasma dessas células ${ }^{1}$. Algumas neoplasias benignas apresentam pleomorfismo celular e hipercromia nuclear, assim como figuras mitóticas, levando muitos autores a acreditar que a presença de invasão óssea ou metástases à distância é necessária para o estabelecimento de um diagnóstico de malignidade ${ }^{1}$.

A avaliação e diagnóstico dos paragangliomas nasais deve incluir tomografia computadorizada, ressonância nuclear magnética e arteriografia com inspeção da fase venosa. A arteriografia também permite a avaliação da circulação colateral. A extensão do tumor e sua relação com as estruturas vasculares do pescoço e base do crânio pode ser estabelecida ${ }^{6}$.

O uso de colorações histológicas especiais visa à demonstração dos grânulos neurossecretores citoplasmáticos ${ }^{3}$. O material para análise histológica pode ser satisfatoriamente obtido por meio de biópsia incisional da lesão, conforme demonstra estudo de Lack et al $^{3}$. Nessa pesquisa, a biópsia incisional propiciou o diagnóstico de todos os casos de paragangliomas nasais (3), carotídeos (3), júgulo-timpânicos (7) e orbital (1), onde foi empregada essa técnica 3 .

Tendo em vista que os paragangliomas apresentam uma tendência à invasão progressiva de estruturas vitais, causando, assim, morbidade, e com a disponibilidade de técnicas cirúrgicas aprimoradas, a excisão cirúgica com margens livres ainda é o tratamento de eleição para essas neoplasias ${ }^{3}$. No entanto, os paragangliomas tendem a apresentar recidiva local em virtude de sua natureza e localização ${ }^{1}$. Há relatos acerca do uso de radioterapia para o tratamento dos paragangliomas, mas os resultados são variáveis. Muitos autores relatam controle adequado com tratamento radioterápico, mas ausência de cura da lesão. Dessa forma, reserva-se esse recurso terapêutico para pacientes que não sejam candidatos à cirurgia ou naqueles com excisão tumoral inadequada. A quimioterapia mostrouse claramente inefetiva no tratamento dos paragangliomas. A embolização tem sido usada primariamente para limitar o volume sangüíneo durante a cirurgia ${ }^{1,8}$.

Paragangliomas podem ocorrer como a síndrome de neoplasia endócrina múltipla (MEN), combinada com carcinoma medular da glândula tireóide, e, facultativamente, feocromocitoma. Nesses casos, a avaliação endocrinológica e ressonância nuclear magnética da região da adrenal, tórax e pescoço são necessárias para uma adequada estratégia terapêutica?.
A incidência de malignidade dos paragangliomas da cabeça e pescoço varia de 4 a 19\%1․ Metástases são raras e ocorrem em, aproximadamente, 9\% dos casos ${ }^{4}$. Relatos da literatura mostram que as metástases dos paragangliomas apresentam propensão para afetar linfonodos, pulmão e ossos ${ }^{2}$. É amplamente aceito que o potencial de malignidade dos paragangliomas não pode ser estimado apenas pelo aspecto histológico dessas lesões ${ }^{2}$. Além de valorizar achados histológicos sugestivos de potencial comportamento maligno, tais como figuras mitóticas, necrose central dos ninhos e invasão vascular, deve-se adotar uma postura cautelosa diante de paragangliomas que demonstram crescimento infiltrativo incomum ou recidivas ${ }^{4,10}$. Tais neoplasias devem ser submetidas a ressecções cirúrgicas agressivas, já que essas características são fortemente sugestivas de comportamento maligno ${ }^{1}$. No entanto, nesses casos específicos, uma intervenção agressiva pode não surtir efeito, pois o prognóstico dos pacientes com paragangliomas malignos é bastante reservado, independentemente da localização da lesão ${ }^{1}$.

\section{COMENTÁRIOS FINAIS}

A ocorrência rara dos paragangliomas nas fossas nasais nos motivou a elaborar esse relato. Uma vez diagnosticados, devem ser tratados cirurgicamente, dada à morbidade que podem ocasionar devido a sua tendência à invasão progressiva de estruturas vitais. No entanto, mesmo com a abordagem cirúrgica adequada, os paragangliomas tendem a apresentar recidiva local em virtude de sua natureza e localização.

\section{REFERÊNCIAS BIBLIOGRÁFICAS}

1. Nguyen Q, Gibbs P, Rice D. Malignant nasal paraganglioma: a case report and review of the literature. Otolaryngology - Head and Neck Surgery 1995; 113(1): 157-61.

2. Talbot A. Paraganglioma of the maxillary sinus. J Laryngol Otol 1990; 104: 248-51.

3. Lack E, Cubilla A, Woodruff J, Farr H. Paragangliomas of the head and neck region: a clinical study of 69 patients. Cancer 1977; 39: 397-409.

4. Battiata B, Vander W, Adair C, Mair E. Quiz case. Archives of Otolaryngology - Head and Neck Surgery 2001; 127(3): 329-30.

5. Himelfarb M, Ostrzega N, Samuel J, Shanon E. Paraganglioma of the nasal cavity. Laryngoscope 1983; 93: 350-2.

6. Mavez M, Carl Rusty. In: Bailey B, Healy G, Johnson J. Head \& Neck Surgery- Otolaryngology, $3^{\text {rd }}$ Edition. Philadelphia: LippincottWilliams \& Wilkins, 2001; p. 1562-73.

7. Kuhn J, Aronoff B. Nasal and nasopharingeal paraganglioma. J Surg Oncol 1989; 40: 38-45.

8. Watson DJ. Nasal paraganglioma. J Laryngol Otol 1988; 102: 526-9.

9. Maier W, Marangos N, Laszig R. J. Laryngol Otol 1999; 113(11): 978-82.

10. Branham G, Gnepp D, O'Menomey S, Friedman W. Malignant paraganglioma - a case report and literature review. Otolaryngol Head Neck Surg 1989; 101: 99-103. 